\title{
High-temperature deformation resistance and creep resistance of a TiAl-based alloy fabricated by cold crucible directional solidification technology
}

\author{
Shu-lin Dong',2, Xin Ding', *Rui-run Chen', Jing-jie Guo', Heng-zhi Fu \\ 1. Key Laboratory of Electromagnetic Processing of Materials (Ministry of Education), Northeastern University, Shenyang 110819, China \\ 2. School of Materials Science and Engineering, Harbin Institute of Technology, Harbin 150001, China
}

\begin{abstract}
In order to improve the high-temperature deformation resistance and creep resistance of TiAl-based alloys, cold crucible directional solidification (CCDS) technology was employed. A $\beta$-type TiAl-based alloy with the nominal composition of Ti44AI6Nb1Cr2V was prepared using the optimized CCDS parameters of $45 \mathrm{~kW}$ input power and $0.5 \mathrm{~mm} \cdot \mathrm{min}^{-1}$ solidification rate. Thermo-compression testing was utilized to evaluate the hightemperature deformation resistance and creep resistance of the CCDS Ti44AI6Nb1Cr2V alloy. Results show that the CCDS Ti44AI6Nb1Cr2V alloy billets contain aligned columnar grains and a high percentage of small-angle lamellae. Thermo-compression testing results in the radial direction of the CCDS alloy show a much higher peak stress than other reported results in similar conditions. The much higher hardening exponent and deformation activation energy are obtained, corresponding to the excellent high-temperature deformation resistance and creep resistance, which are because of the hard-oriented grains, weaker stress-strain coordination capability of lamella structure and relatively more hysteretic dynamic recrystallization. Thermo-compression testing results in the longitudinal direction of the CCDS Ti44AI6Nb1Cr2V alloy show the much higher peak stress than that in the radial direction, indicating the better high-temperature deformation resistance and creep resistance attributed to the hard-oriented lamellae in this condition.
\end{abstract}

Key words: cold crucible directional solidification; TiAl; thermo-compression; constitutive equations; microstructure CLC numbers: TG146.23 Document code: A

Article ID: 1672-6421(2020)05-378-06

$\mathrm{T}$ iAl-based alloys have wide application prospect in the aerospace field due to their low density, high strength and high service temperature ${ }^{[1-2]}$. As the potential high-temperature structural materials, the higher high-temperature deformation resistance and creep resistance are of importance ${ }^{[3-5]}$, which are directly related with the service capability and service life of workpieces.

Cold crucible directional solidification (CCDS) technology is an excellent fabrication method to improve the high-temperature deformation resistance and creep resistance of TiAl-based alloys ${ }^{[6-7]}$, since the aligned columnar grains and aligned $\left(\alpha_{2}+\gamma\right)$ lamellae can be obtained by using this method. The aligned columnar grains can be easily obtained by CCDS, because stable and sufficient power can be supplied for the directional growth of grains during directional solidification ${ }^{[8,9]}$.

\section{*Rui-run Chen}

Male, Ph.D., Professor. Research interests: high-temperature alloys, high-entropy alloys and energy storage materials. He has published more than 200 papers.

E-mail: ruirunchen@hit.edu.cn

Received: 2020-06-16; Accepted: 2020-08-11
To obtain the aligned $\left(\alpha_{2}+\gamma\right)$ lamellae by CCDS, $\beta$-type TiAl-based alloys should be chosen ${ }^{[10]}$. In that way, the primary $\beta$ phase grows with $\{100\}_{\beta}$ along the directional solidification direction ${ }^{[11]}$. Theoretically, because of the orientation relationship of $\{110\}_{\beta} / /\{0001\}_{\alpha} / /\{111\}_{\gamma}{ }^{[14]}$, there should be $1 / 3$ small-angle lamellae $\left(0^{\circ}-30^{\circ}\right.$ lamellae) in the final microstructure. However, CCDS technology can produce a relatively higher temperature gradient for the large-sized alloy billet, accompanied with a lower solidification rate, which can provide more energy for the nucleation and growth of $\left(\alpha_{2}+\gamma\right)$ lamellae, promoting the formation of more small-angle lamellae ${ }^{[13]}$. Previous studies indicated that, by using $\beta$-type TiAlbased alloys and optimized CCDS parameters, the percentage of small-angle lamellae could reach more than $60 \%{ }^{[10]}$

It is difficult to evaluate the high-temperature deformation resistance and creep resistance of CCDS alloys, which will take a long time for the testing. In this study, a novel and effective way, i.e., the thermocompression testing on Gleeble (Dynamic Systems, Inc., USA), was employed to evaluate the hightemperature deformation resistance and creep resistance 
of the alloy. The thermo-compression constitutive equations of the CCDS TiAl-based alloys are established, where the value of the hardening exponent $(n)$ and the deformation activation energy $(Q)$ can be obtained. On the basis of existing results ${ }^{[4,14-17]}$, the higher the value of hardening exponent and deformation activation energy, the more excellent the high-temperature deformation resistance ${ }^{[16]}$ and creep resistance ${ }^{[17]}$. In addition, via studying the microstructure of thermo-compression samples, the reasons why the CCDS TiAl-based alloys yield the excellent performances can be understood.

A $\beta$-type Ti44Al6Nb1Cr2V (at.\%) alloy is chosen in this study. This alloy has a modified near lamellar structure, containing a high percentage of $\left(\alpha_{2}+\gamma\right)$ lamellae in the as-cast condition ${ }^{[1,10]}$. The optimized CCDS parameters ${ }^{[8,10]}$ are used to fabricate the alloy, with the aim of acquiring aligned columnar grains and a higher percentage of small-angle $\left(\alpha_{2}+\gamma\right)$ lamellae. Thermo-compression testing was carried out on the CCDS Ti44A16Nb1Cr2V alloy billets in both radial and longitudinal directions to evaluate the high-temperature deformation resistance and creep resistance. The temperature of $1,100{ }^{\circ} \mathrm{C}-1,250{ }^{\circ} \mathrm{C}$ and strain rate of $0.001 \mathrm{~s}^{-1}-1.0 \mathrm{~s}^{-1}$ were chosen in the thermocompression testing, and the experimental data obtained in this range can be employed to establish the thermo-compression constitutive equations on the basis of the hyperbolic sine function and Arrhenius mathematical model ${ }^{[4,14]}$. From the constitutive equations, the hardening exponent and deformation activation energy can be obtained, which can be used to evaluate the high-temperature deformation resistance and creep resistance of the CCDS Ti44Al6Nb1Cr2V alloy ${ }^{[16,17]}$. The microstructure of the thermo-compression samples is also investigated to explain the enhanced high-temperature deformation resistance and creep resistance of the CCDS Ti44A16Nb1Cr2V alloy.

\section{Experiment procedure}

A Master ingot of Ti44A16Nb1Cr2V alloy with the size of about $225 \mathrm{~mm}$ in diameter and $320 \mathrm{~mm}$ in height was prepared using high-purity Ti, Al alloy, and Al-Nb, Al-Cr, and Al-V master alloys by vacuum consumable melting. The master ingot was cut into cylindrical feeding bars of $19 \mathrm{~mm}$ in diameter and 100-320 mm long for CCDS fabrication.

The CCDS apparatus comprises the heating system, water cooling system, vacuum system, feeding/drawing system and control system. During CCDS, the crucible, Ga-In container, coil and furnace inner wall were cooled by the high-pressure circulating water. The feeding bar and drawing bar in the coil were melted by the high-frequency eddy current. The molten alloy was continuously drawn to the Ga-In liquid metal. The relatively higher temperature gradient can be created between the high-temperature molten alloy and the low-temperature GaIn liquid metal. The optimized CCDS parameters of $45 \mathrm{~kW}$ input power and $0.5 \mathrm{~mm} \cdot \mathrm{min}^{-1}$ solidification rate were used to fabricate the alloy billets ${ }^{[1,7,10]}$, and the temperature gradient at the liquid/solid interface was about $21.0 \mathrm{~K} \cdot \mathrm{mm}^{-1}{ }^{[18]}$. The whole heating process was carried out in the $300 \mathrm{~Pa}$ high- purity argon atmosphere. The drawing distance was about 70 $\mathrm{mm}$. The composition determined by chemical analysis in the directional solidification (DS) zone of the alloy billet is Ti43.68A15.65Nb0.91Cr1.79V.

The obtained CCDS billets were used for the thermocompression testing on Gleeble 1500D both in radial and longitudinal directions. Here, the radial direction (RD) refers to the compressive direction being perpendicular to the DS direction, and the longitudinal (LD) refers to the compressive direction being parallel with the DS direction. The dimensions of the thermocompression samples are $8 \mathrm{~mm}$ in diameter and $12 \mathrm{~mm}$ in height. For the radial testing, the temperatures $(T)$ of $1,100^{\circ} \mathrm{C}, 1,150{ }^{\circ} \mathrm{C}$, $1,200{ }^{\circ} \mathrm{C}, 1,250{ }^{\circ} \mathrm{C}$, and the strain rates $(\dot{\varepsilon})$ of $0.001 \mathrm{~s}^{-1}, 0.01 \mathrm{~s}^{-1}$, $0.1 \mathrm{~s}^{-1}, 1.0 \mathrm{~s}^{-1}$ were used; for the longitudinal testing, the parameters of $1,150{ }^{\circ} \mathrm{C} / 0.01 \mathrm{~s}^{-1}, 1,200{ }^{\circ} \mathrm{C} / 0.1 \mathrm{~s}^{-1}, 1,200{ }^{\circ} \mathrm{C} /$ $0.01 \mathrm{~s}^{-1}, 1,200{ }^{\circ} \mathrm{C} / 0.001 \mathrm{~s}^{-1}, 1,250{ }^{\circ} \mathrm{C} / 0.01 \mathrm{~s}^{-1}$ were used. The compressive engineering strain is $50 \%$ (about 0.69 true strains). A Pt-Rh thermocouple was used to trace the temperature of thermo-compression samples. After compressing, the samples were cut along longitudinal sections, and polished for microstructure investigation.

The microstructures of the master ingot and CCDS alloy billets were observed and analyzed using an optical microscope (OM). The microstructures of thermo-compression samples were detected by scanning electron microscopy (SEM) in backscattered electron (BSE) mode and electron back-scattered diffraction (EBSD) mode. The OM, SEM and EBSD samples were all firstly treated by a standard metallographic method. The OM samples were then etched by a reagent composed of $10 \%$ $\mathrm{HF}, 2 \% \mathrm{HNO}_{3}$ and $88 \% \mathrm{H}_{2} \mathrm{O}$ (volume ratio); SEM samples were not etched; EBSD samples were electrochemically polished using a modified Kroll's reagent ${ }^{[12]}$ at $15-20 \mathrm{~V}$ and $-25^{\circ} \mathrm{C}$.

\section{Results and discussion}

Figure 1 shows the picture and microstructures of the Ti44A16Nb1Cr2V master ingot. The ingot has a modified nearlamellar structure, containing lots of $\left(\alpha_{2}+\gamma\right)$ lamellae and some $(\mathrm{B} 2+\gamma)$ blocky morphology ${ }^{[1,10]}$. Microcracks can be observed in the microstructure. The formation of microcracks is generally related to the internal stress and intrinsic brittleness of the alloy. In the brittle TiAl-based alloys, the microcracks will rapidly propagate along lamellae and grain boundaries under stress, forming large crack, and therefore, leading to the poor mechanical properties. A previous study showed that, at room temperature, the average ultimate tensile strength was around $420 \mathrm{MPa}$, and the average tensile elongation was about $0.40 \%$ for this master alloy ${ }^{[19]}$.

Figure 2(a) shows the surface morphology of a CCDS billet fabricated at $45 \mathrm{~kW}$ input power and $0.5 \mathrm{~mm} \cdot \mathrm{min}^{-1}$ solidification rate. This billet can be divided into three zones from the top to bottom, i.e., liquid zone, DS zone and bottom zone. The samples for the thermo-compression testing were obtained from the DS zone. Figure 2(b) shows the microstructure in the DS zone, where the inserts are the magnified images. There are 

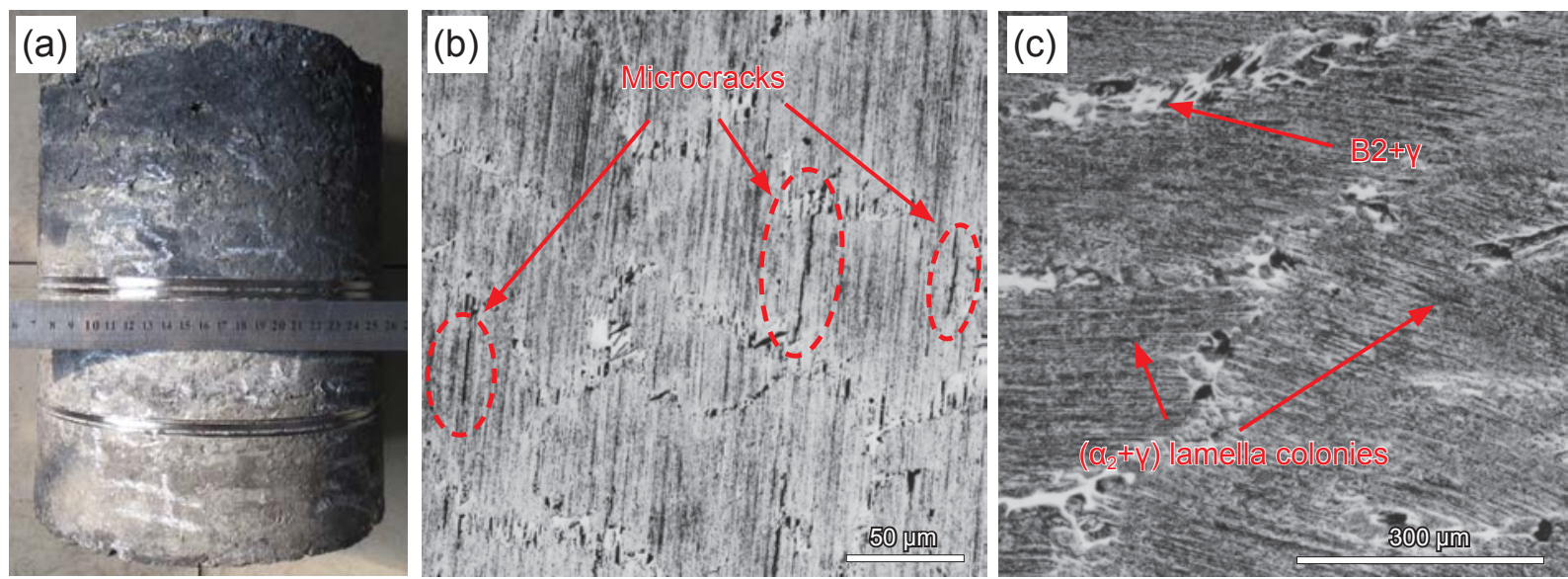

Fig. 1: Master ingot (a) and microstructure (b, c) of Ti44AI6Nb1Cr2V alloy
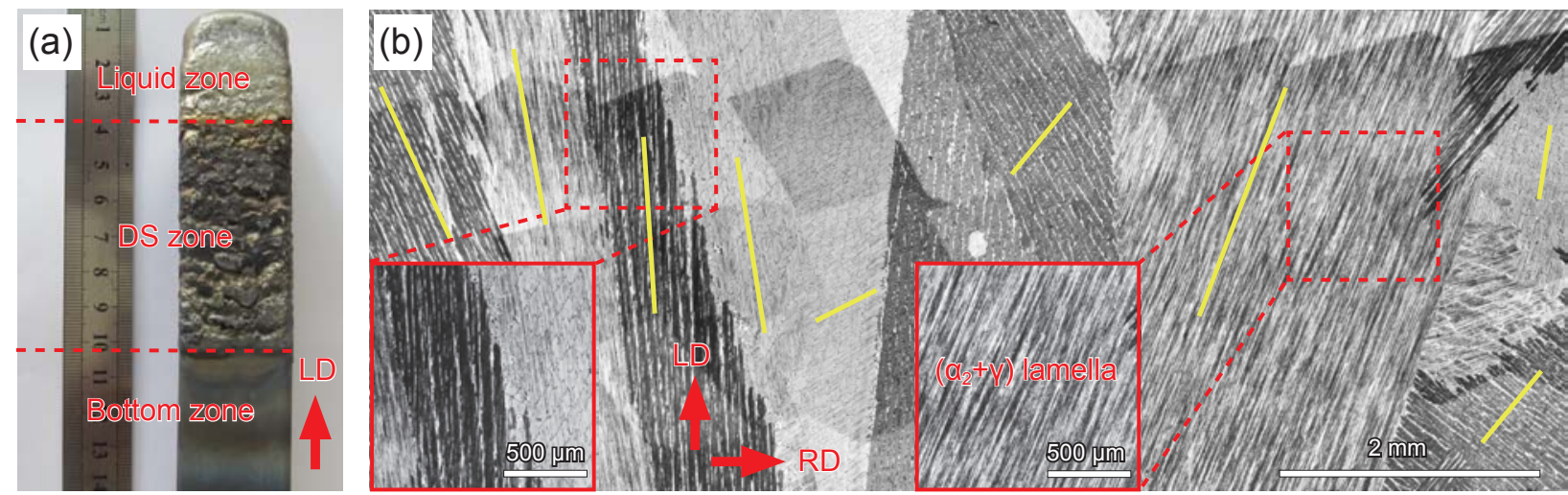

Fig. 2: Surface morphology of CCDS alloy billet prepared at $45 \mathrm{~kW}$ input power and $0.5 \mathrm{~mm} \cdot \mathrm{min}^{-1}$ solidification rate (a); OM microstructure in DS zone (b)

almost no defects in the microstructure. The aligned columnar grains and lots of small-angle lamellae can be observed. The percentage of small-angle lamellae for the whole alloy billet can reach about $62 \%{ }^{[10]}$. As discussed above, the aligned columnar grains and higher percentage of small-angle lamellae can be attributed to the CCDS technological characteristics, and the optimized combination of temperature gradient and solidification rate.

Figure 3 shows the true stress-strain curves obtained from radial thermo-compression testing at strain rates of $0.001 \mathrm{~s}^{-1}$, $0.01 \mathrm{~s}^{-1}, 0.1 \mathrm{~s}^{-1}, 1.0 \mathrm{~s}^{-1}$, and temperatures of $1,100{ }^{\circ} \mathrm{C}, 1,150{ }^{\circ} \mathrm{C}$, $1,200{ }^{\circ} \mathrm{C}, 1,250{ }^{\circ} \mathrm{C}$. It can be found that, with the increase of the true strain, the true stress firstly increases rapidly, and then decreases slowly after reaching a peak value. In addition, the compressed CCDS alloy shows a higher true stress and greater strain rate at lower temperature. For the radial thermocompression, the pressure is perpendicular to the aligned columnar grains, therefore, the columnar grains are the hard-oriented grains. Moreover, the near-lamellar structure containing lots of lamellae yields a weaker stress-strain coordination capability. Consequently, the CCDS alloy exhibits a much higher peak stress in radial thermo-compression testing than other reported results in similar conditions ${ }^{[4,5]}$.

The hyperbolic sine function and Arrhenius mathematical model ${ }^{[20]}$ were employed to establish the constitutive equations using the results of peak stress, by which the value of hardening exponent $(n)$ and deformation activation energy $(Q)$ can be obtained. Eight times of iterations that involve the relationship of peak stress $\left(\sigma_{\mathrm{p}}\right)$, strain rate $(\dot{\varepsilon})$, temperatures $(T)$ and ZenerHollomon $(Z)$ parameter were employed for more accurate results. Finally, the thermo-compression constitutive equations in radial direction for the CCDS Ti44Al6Nb1Cr2 V alloy in the DS zone are obtained as follows:

$$
\begin{gathered}
\dot{\varepsilon}=2.66 \times 10^{24} \cdot\left[\sinh \left(0.003703 \sigma_{\mathrm{p}}\right)\right]^{3.90} \cdot \exp \left(-\frac{729600}{R T}\right) \\
\sigma_{\mathrm{p}}=\frac{1}{0.003703} \ln \left\{\left(\frac{Z}{2.66 \times 10^{24}}\right)^{\frac{1}{3.90}}+\right. \\
{\left[\left(\frac{Z}{2.66 \times 10^{24}}\right)^{\left.\left.\frac{2}{3.90}+1\right]^{\frac{1}{2}}\right\}}\right.}
\end{gathered}
$$

where $R$ is the gas constant. The value of hardening exponent $(n=3.90)$ is much higher than other reported results ${ }^{[5,23]}$, denoting an excellent high-temperature deformation resistance of this CCDS alloy in radial direction. The value of deformation activation energy $\left(Q=729.6 \mathrm{~kJ} \cdot \mathrm{mol}^{-1}\right)$ is also much higher than other reported results ${ }^{[20,21]}$, denoting an excellent creep resistance in radial direction as mentioned above.

Figure 4 shows the microstructure of compressed samples by SEM-BSE and EBSD after radial thermo-compression at $1,100^{\circ} \mathrm{C}$ $/ 1.0 \mathrm{~s}^{-1}, 1,150{ }^{\circ} \mathrm{C} / 0.1 \mathrm{~s}^{-1}, 1,200{ }^{\circ} \mathrm{C} / 0.01 \mathrm{~s}^{-1}$ and $1,250{ }^{\circ} \mathrm{C}$ 

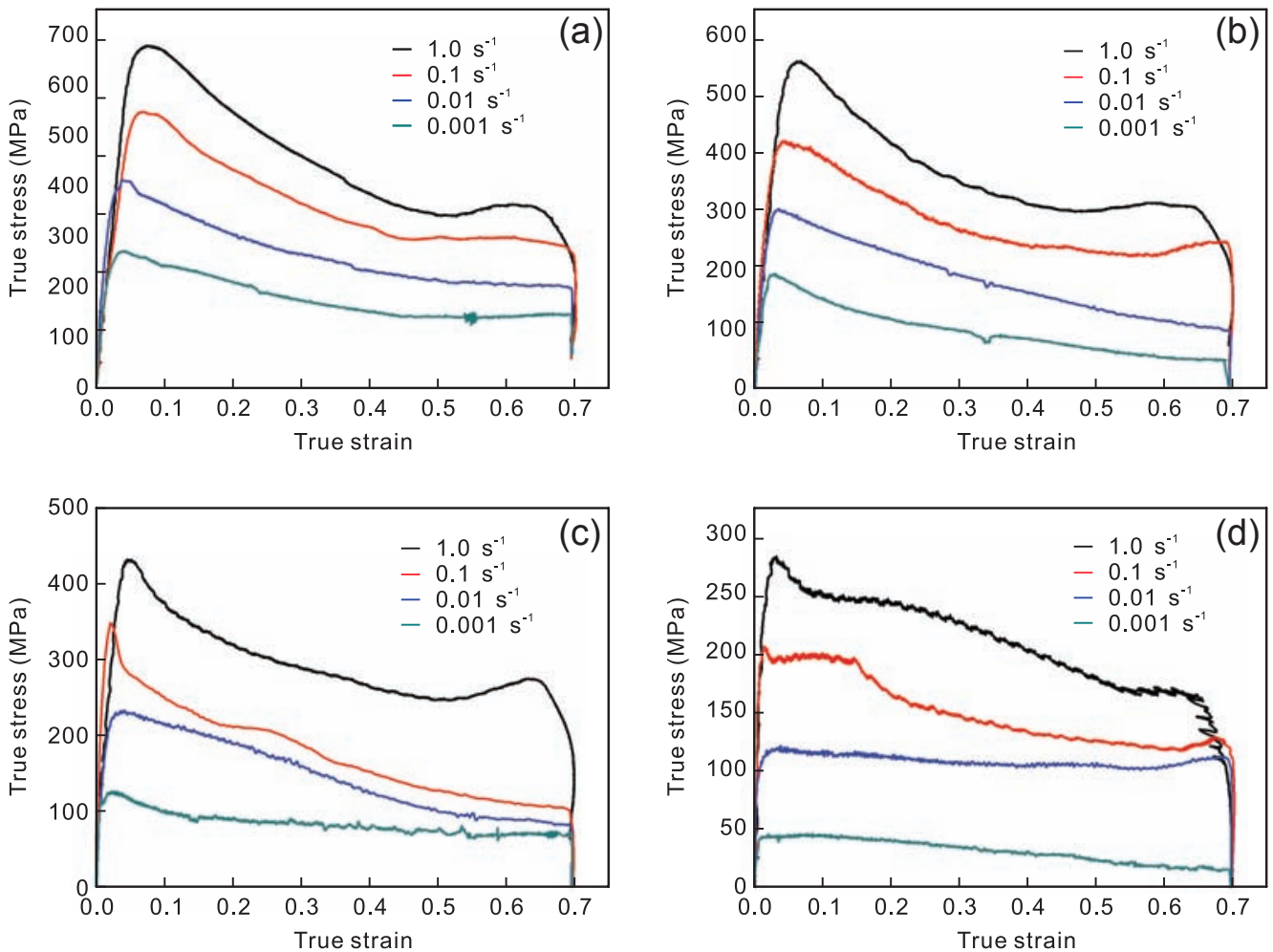

Fig. 3: True stress-strain curves of CCDS Ti44AI6Nb1Cr2V alloy obtained from radial thermo-compression testing at strain rates of $1.0 \mathrm{~s}^{-1}, 0.1 \mathrm{~s}^{-1}, 0.01 \mathrm{~s}^{-1}, 0.001 \mathrm{~s}^{-1}$ under different temperatures: (a) $1,100{ }^{\circ} \mathrm{C}$;

(b) $1,150{ }^{\circ} \mathrm{C}$; (c) $1,200^{\circ} \mathrm{C}$; (d) $1,250^{\circ} \mathrm{C}$

$/ 0.001 \mathrm{~s}^{-1}$, respectively. Figure 4(a)-(d) shows the SEM-BSE images at the lower magnification times. The fiber structure can be observed in all samples. The direction of fiber structure is nearly perpendicular to the compressive direction.

Figure 4(e)-(h) are the EBSD results at higher magnification levels, including grain orientation spread (GOS) figures, grain boundaries (GBs), and $\{001\}$ pole figures of $\gamma$ phase. GOS means the average difference in orientation between the average grain orientation and all measurements in one grain ${ }^{[22]}$. Utilizing GOS figures, the dynamic recrystallization (DRX)

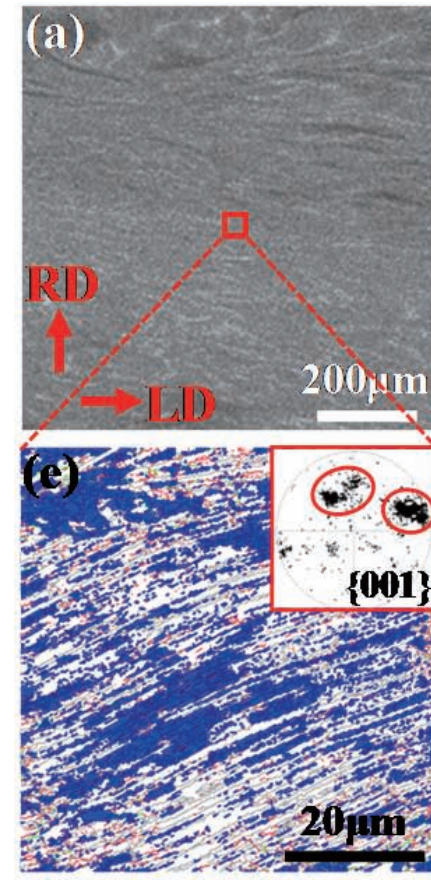

$2^{\circ} \leq x \leq 5^{\circ}$ low -angle GBs

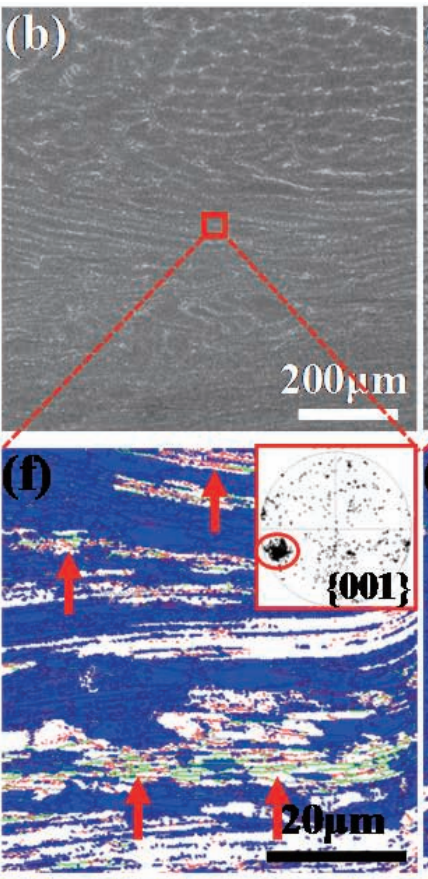

$5^{\circ}<\mathrm{y} \leq 15^{\circ}$ medium-angle GBs

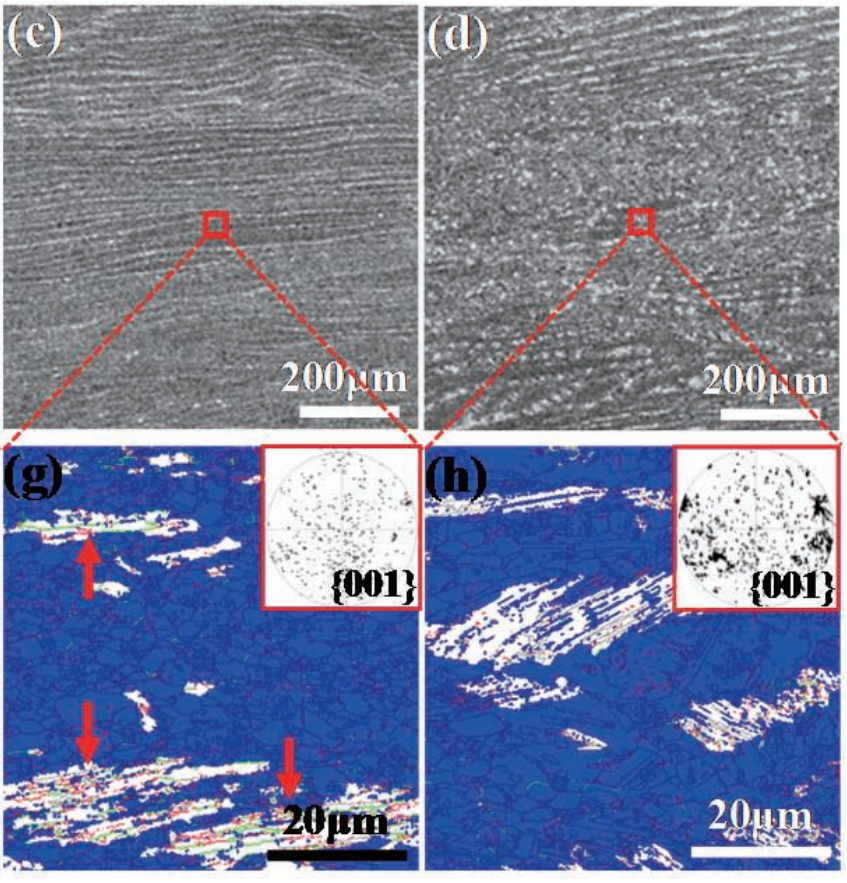

$15^{\circ}<\mathrm{z} \leq 180^{\circ}$ high-angle GBs

Fig. 4: SEM-BSE microstructure at $1,100^{\circ} \mathrm{C} / 1.0 \mathrm{~s}^{-1}$ (a), $1,150{ }^{\circ} \mathrm{C} / 0.1 \mathrm{~s}^{-1}$ (b), $1,200{ }^{\circ} \mathrm{C} / 0.01 \mathrm{~s}^{-1}$ (c) and $1,250{ }^{\circ} \mathrm{C} / 0.001 \mathrm{~s}^{-1}(\mathrm{~d})$, respectively; and corresponding EBSD (e-h) results including GOS figures, GBs, and $\{001\}$ pore figures of $\mathrm{Y}$ phase after radial thermo-compression testing 
percentage can be quantified on the basis of the GOS value of DRX grains being lower than that of deformed grains. It has been confirmed that, those regions yielding the GOS value less than $2.1^{\circ}$ are the DRX grains ${ }^{[16]}$, i.e., the blue regions in Fig. 4 are the DRX grains. The DRX percentage is $50.0 \%$, $62.1 \%, 81.3 \%$ and $84.1 \%$ respectively at the four groups of compressive parameters. The white regions are deformed grains, corresponding to the residual lamellae. In the residual lamellae, there are lots of low-angle and medium-angle GBs that are related to the active dislocation behavior in these regions. The poles in the inserts are more concentrated at $1,100{ }^{\circ} \mathrm{C} / 1.0 \mathrm{~s}^{-1}$ and $1,150{ }^{\circ} \mathrm{C} / 0.1 \mathrm{~s}^{-1}$, because there is higher percentage of residual lamellae that originally belongs to one or few lamella colonies. The poles are more dispersive at $1,200{ }^{\circ} \mathrm{C} / 0.01 \mathrm{~s}^{-1}$, because the non-oriented DRX grains appear instead of residual lamellae. The poles are also dispersive at $1,250{ }^{\circ} \mathrm{C} / 0.001 \mathrm{~s}^{-1}$, and the pole density becomes higher, because the DRX grains are coarsened in this condition.

Via the microstructure investigation, it can be concluded that the fiber structure comprises the deformed residual lamellae and DRX grains when the value of Zener-Hollomon parameters is higher, such as $1,100{ }^{\circ} \mathrm{C} / 1.0 \mathrm{~s}^{-1}$ and $1,150^{\circ} \mathrm{C} / 0.1 \mathrm{~s}^{-1}$ [Figs. $4(\mathrm{e})$ and (f)]; the fiber structure comprises a small quantity of deformed residual lamellae and more (coarsened) DRX grains at lower $\mathrm{Z}$ parameters such as $1,200{ }^{\circ} \mathrm{C} / 0.01 \mathrm{~s}^{-1}$ and $1,250{ }^{\circ} \mathrm{C} / 0.001 \mathrm{~s}^{-1}$ [Figs. 4(g) and (h)]. This CCDS alloy has the relatively more hysteretic DRX than other reported results ${ }^{[4,5,21,23]}$ in similar conditions, which is also a factor contributing to the higher high- temperature deformation resistance and creep resistance.

The mechanical properties in longitudinal direction (i.e., DS direction) of the alloy can be strongly enhanced by CCDS. To evaluate the high-temperature deformation resistance and creep resistance in the longitudinal direction, the thermocompression testing in this direction has also been carried out. The derivation process and analysis methods for obtaining the constitutive equations in the longitudinal direction is the same as that in the radial direction. Thus, merely five groups of thermo-compression parameters in the longitudinal direction were employed, which also have been applied in radial testing. Figure 5(a) shows the true stress-strain curves compressed at different parameters. Figure 5(b) shows the comparison of the peak stress in the longitudinal direction and radial direction at the five groups of compressive parameters. The difference values of peak stress in the two compressive directions are given beside the double-headed arrows. The longitudinal peak stress is much higher than the radial peak stress, which is due to, not only the hard-oriented grains, weaker stressstrain coordination capability of lamella structure and more hysteretic DRX, but also the high percentage of lowangle lamellae, which are the hard-oriented lamellae in this condition. According to the discussion in radial direction, the much higher longitudinal peak stress implies the more excellent high-temperature deformation resistance and creep resistance in this direction. Furthermore, it also means that the CCDS Ti44Al6Nb1Cr2V alloy yields the much better mechanical properties in the DS direction.
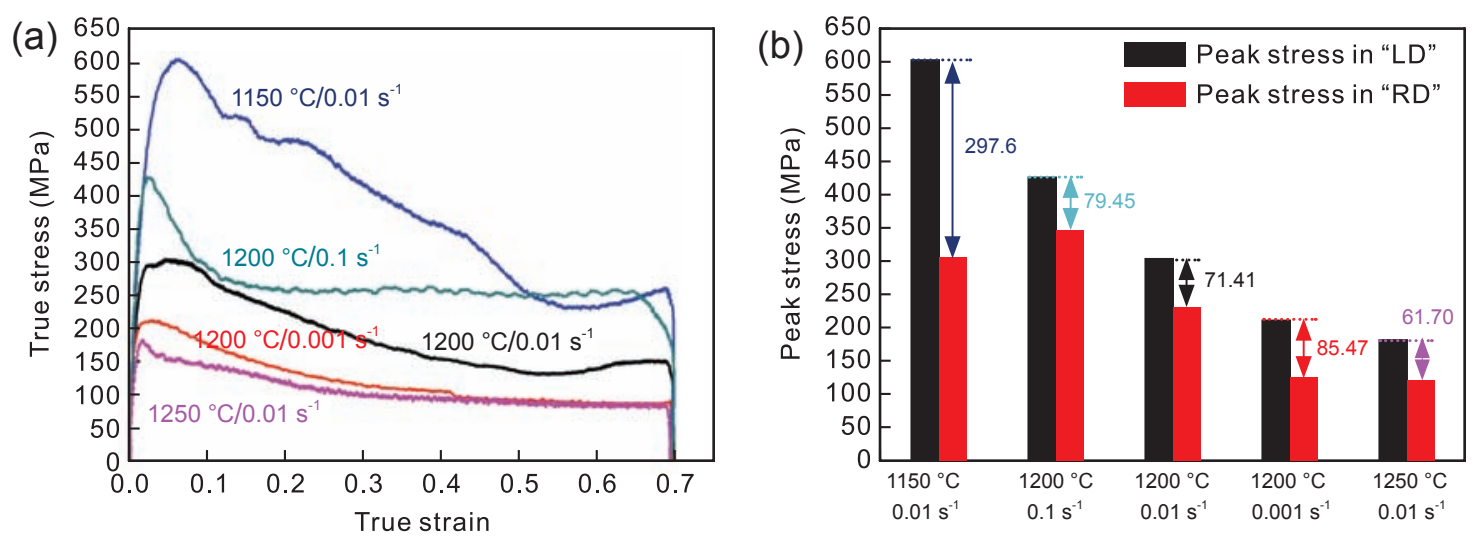

Fig. 5: True stress-strain curves of CCDS Ti44AI6Nb1Cr2V alloy obtained from longitudinal thermo-compression testing compressed at $1,150^{\circ} \mathrm{C} / 0.01 \mathrm{~s}^{-1}, 1,200^{\circ} \mathrm{C} / 0.1 \mathrm{~s}^{-1}, 1,200^{\circ} \mathrm{C} / 0.01 \mathrm{~s}^{-1}, 1,200^{\circ} \mathrm{C} / 0.001 \mathrm{~s}^{-1}$ and $1,250^{\circ} \mathrm{C} / 0.01 \mathrm{~s}^{-1}$ (a), and bar graph exhibiting the peak stress in longitudinal and radial directions compressed at five groups of parameters (b)

\section{Conclusions}

Cold crucible directional solidification (CCDS) technology is utilized to fabricate the $\beta$-type Ti44A16Nb1Cr2 $\mathrm{V}$ alloy at $45 \mathrm{~kW}$ input power and $0.5 \mathrm{~mm} \cdot \mathrm{min}^{-1}$ solidification rate. The microstructure composed of aligned columnar grains and high percentage of low-angle lamellae was obtained. Thermocompression testing is used to evaluate the high-temperature deformation resistance and creep resistance of the alloy. Following conclusions are obtained:
(1) The CCDS Ti44A16Nb1Cr2V alloy shows the higher peak stress in the thermo-compression testing in radial direction. The thermo-compression constitutive equations are established. The much higher hardening exponent and deformation activation energy are obtained, corresponding to the excellent high-temperature deformation resistance and creep resistance of the alloy, as a result of the hard-oriented grains and weaker stress-strain coordination capability of lamella structure, as well as the relatively more hysteretic dynamic recrystallization. 
(2) The peak stress in longitudinal direction is much higher than that in radial direction, indicating a much better hightemperature deformation resistance and creep resistance in this direction, due to the higher percentage of low-angle hardoriented lamellae in this condition.

\section{Acknowledgements}

This research was supported by the National Natural Science Foundation of China (Grant Nos. 51825401 and 51601034), and the Fundamental Research Funds for the Central Universities (Grant No. N180904005).

\section{References}

[1] Chen R R, Dong S L, Guo J J, et al. Investigation of macro/ microstructure evolution and mechanical properties of directionally solidified high-Nb TiAl-based alloy. Materials and Design, 2016, 89: 492-506.

[2] Cen M J, Liu Y, Chen X, et al. Inclusions in melting process of titanium and titanium alloys. China Foundry, 2019, 16(4): 223231.

[3] Chen G, Peng Y B, Zheng G, et al. Polysynthetic twinned TiAl single crystals for high-temperature applications. Nature Materials, 2016, 15(8): 876-881.

[4] Niu H Z, Chen Y Y, Xiao S L, et al. High temperature deformation behaviors of Ti-45Al-2Nb-1.5V-1Mo-Y alloy. Intermetallics, 2011, 19(2): 1767-1774.

[5] Singh V, Mondal C, Kumar A, et al. High temperature compressive flow behavior and associated microstructural development in a $\beta$-stabilized high Nb-containing $\mathrm{y}$-TiAl based alloy. Journal of Alloys and Compounds, 2019, 788: 573-585.

[6] Zhao J, Zhang Z Y, Liu S B, et al. Elimination of misrun and gas hole defects of investment casting TiAl alloy turbocharger based on numerical simulation and experimental study. China Foundry, 2020, 17(1): 29-34.

[7] Chen R R, Wang Q, Zhou Z C, et al. Microstructure, tensile properties and creep behavior of high-Al TiAINb alloy using electromagnetic cold crucible continuous casting. Journal of Alloys and Compounds, 2019, 801: 166-174.

[8] Yang J R, Chen R R, Su Y Q, et al. Optimization of electromagnetic energy in cold crucible used for directional solidification of TiAl alloy. Energy, 2018, 161: 143-155.

[9] Kim S E, Lee Y T, Oh M H, et al. Directional solidification of TiAlSi alloys using a polycrystalline seed. Intermetallics, 2000, 8(4): 399-405.
[10] Dong S L, Chen R R, Guo J J, et al. Microstructure control and mechanical properties of Ti44Al6Nb1.0Cr2.0V alloy by cold crucible directional solidification. Materials Science and Engineering: A, 2014, 614: 67-74.

[11] Ding X F, Lin J P, Zhong L Q, et al. Microstructural control of TiAl-Nb alloys by directional solidification. Acta Materialia, 2012, 60(2): 498-506.

[12] Jun I S, Jang H S, Oh M H, et al. Microstructure control of TiAl alloys containing $\beta$ stabilizers by directional solidification. Materials Science and Engineering: A, 2002, 329-331: 13-18.

[13] Jung I S, Oh M H, Park N J, et al. Lamellar boundary alignment of DS-processed TiAI-W alloys by a solidification procedure. Metals and Materials International, 2007, 13(6): 455-462.

[14] Dong S L, Liu T, Li Y J, et al. Hot deformation processing capability of Fe-contained high Nb TiAl-based alloy. Vacuum, 2019, 159: 391-399.

[15] Appel F. Diffusion assisted dislocation climb in intermetallic gamma TiAl. Materials Science and Engineering: A, 2001, 317(1-2): 115-127.

[16] Dong S L, Chen R R, Guo J J, et al. Deformation behavior and microstructural evolution of directionally solidified TiAINb-based alloy during thermo-compression at 1,373-1,573 K. Materials and Design, 2015, 84: 118-132.

[17] McQueen H J, Ryan N D. Constitutive analysis in hot working. Materials Science and Engineering: A, 2002, 322(1-2): 43-63.

[18] Chen R R, Dong S L, Guo J J, et al. Microstructure evolution and mechanical properties of directionally-solidified TiAINb alloy in different temperature gradients. Journal of Alloys and Compounds, 2015, 648: 667-675.

[19] Dong S L, Chen R R, Guo J J, et al. Microstructure and room temperature tensile property of as-cast Ti44AI6Nb1.0Cr2.0V alloy. Transactions of Nonferrous Metals Society of China, 2015, 25(4): 1097-1105.

[20] Prasad Y V R K, Seshacharyulu T. Modelling of hot deformation for microstructural control. International Materials Reviews, 1998, 43(6): 242-258.

[21] Rao K P, Prasad Y V R K. Processing map and hot working mechanisms in a P/M TiAl alloy composite with in situ carbide and silicide dispersions. Materials Science and Engineering: $A$, 2010, 527(24-25): 6589-6595.

[22] Gao Y, Di H S, Zhang J Q, et al. An electron backscattered diffraction study on the dynamic recrystallization behavior of a nickel-chromium alloy $(800 \mathrm{H})$ during hot deformation. Materials Science and Engineering: A, 2013, 585: 71-85.

[23] Godor F, Werner R, Lindemann J, et al. Characterization of the high temperature deformation behavior of two intermetallic TiAlMo alloys. Materials Science and Engineering: A, 2015, 648: 208-216. 